\title{
Surface Roughness Imparts Tensile Ductility to Nanoscale Metallic Glasses
}

Sara Adibi, ${ }^{1,2}$ Paulo S. Branicio, ${ }^{2}$ Rachel Liontas, ${ }^{3}$ David Z. Chen, ${ }^{4}$ Julia R. Greer, ${ }^{4}$ David J.

\author{
Srolovitz, ${ }^{5}$ and Shailendra P. Joshi ${ }^{1}$
}

${ }^{1}$ Department of Mechanical Engineering, National University of Singapore, 117576, Singapore

${ }^{2}$ Institute of High Performance Computing, A*STAR, 138632, Singapore

${ }^{3}$ Division of Chemistry and Chemical Engineering, California Institute of Technology, Pasadena, CA 91125 USA

${ }^{4}$ Division of Engineering and Applied Science, California Institute of Technology, Pasadena, CA 91125 USA

${ }^{5}$ Deptartments of Materials Science and Engineering \& Mechanical Engineering and Applied Mechanics, University of Pennsylvania, Philadelphia, PA 19104 USA

\begin{abstract}
:
Experiments show an intriguing brittle-to-ductile transition on size reduction on nanoscale metallic glasses (MGs). Here we demonstrate that such phenomena is linked to a fundamental characteristic size effect in the failure mode under tensile loading. Large-scale molecular dynamics simulations reveal that nanoscaled MGs with atomistically smooth surface exhibit catastrophic failure via sharp, localized shear band propagation. In contrast, nanosized specimens with surface imperfections exhibit a clear transition from shear banding to necking instability above a critical roughness ratio of $\xi \sim 1 / 20$, defined as the ratio between the average surface imperfection size and sample diameter. The observed brittle-to-ductile transition that emerges in nanosized MGs deformed at room temperature can be strongly attributed to this roughness argument. In addition, the results suggest that the suppression of brittle failure may be scale-free and be realizable on length scales much beyond those considered here, provided the threshold roughness ratio is exceeded. The fundamental critical roughness ratio demonstrated sheds light on the complex mechanical behavior of amorphous metals and has implications for the application of MGs in nano- and micro-devices.
\end{abstract}

KEYWORDS: Metallic glasses, tensile ductility, Brittle-to-ductile transition, size effect, surface roughness, molecular dynamics simulation.

Bulk metallic glasses (BMGs) represent an attractive class of materials for their outstanding mechanical properties, including high strength, high elastic limit, and high hardness compared with their crystalline counterparts ${ }^{1,2,3}$. A serious limitation of most monolithic BMGs is that they fail catastrophically when subjected to uniaxial tension with little macroscopic plasticity $2,3,4,5$. This is unlike their crystalline analogs that commonly exhibit significant plastic deformation 
prior to failure. The basic carriers of plastic deformation in metallic glasses (MGs) are shear transformation zones (STZs), and catastrophic brittle failure of BMGs is associated with the collective dynamics of STZs that coalesce to form nanoscale shear bands (SBs) in which intense local plastic strain occurs. In macroscale specimens, strategies proposed to mitigate the brittle failure of MGs and induce tensile ductility involve promoting heterogeneous stress states to control SB propagation. For example, carefully designed BMG composites have been shown to produce a ductile response under tensile loading much like crystalline metals ${ }^{6}$. Inducing stress gradients through specimen geometry modification is another approach that increases ductility ${ }^{7}$, ${ }^{8}$. A third strategy to enhance both the deformability and work hardening of MGs is via reduction of the external dimensions of the samples down to the nanometer range ${ }^{9,10,11}$. Experiments on nanoscale samples have shown brittle failure via localized SBs and ductile deformation via necking $2,6,9,10,11,12,13,14,15,16$. A brittle-to-ductile transition (BDT) has been reported to occur at a critical sample size, which is a function of the specific processing steps and chemistry of the glass. A catastrophic SB typically characterizes brittle failure above a critical specimen dimension $d_{c}$, while necking induced plasticity is evident below $d_{c}$ for various MG compositions ${ }^{9}, 10,11,16$. In situ tensile experiments by Guo et al. ${ }^{9}$ indicate necking of monolithic MG samples with sizes in the order of $\sim 100 \mathrm{~nm}$. Jang and Greer ${ }^{10}$ reported extendibility in excess of $20 \%$ and necking in freestanding Zr-based MG nano-samples with diameters of $d \sim 100 \mathrm{~nm}$, while larger diameter specimens failed catastrophically via SBs. Tian et al. ${ }^{11}$ reported a similar transition at $d \sim 80 \mathrm{~nm}$ in nanoscale tensile experiments on freestanding $\mathrm{Cu}_{49} \mathrm{Zr}_{51} \mathrm{MG}$ samples. Several other recent investigations demonstrate clearly ductility in MG nano-pillars fabricated both by electroplating into a template and focused ion beam (FIB) milling ${ }^{16,17,18}$.

These nanoscale experiments were performed on nominally monolithic materials with nominally uniform specimen geometries. It has been suggested that the traction-free surface as well as the surface energy state may play a role in the ductility observed at the nanoscale ${ }^{14,16,19}$, 20, 21 . Previous molecular dynamics (MD) simulations ${ }^{17}$ suggest that surface STZs may initiate with a lower activation barrier compared to those in bulk MGs; this may result in size dependent response on the nanoscale ${ }^{17}$. The caveat is that the sample size at which the ductility transition occurs in atomistic simulations is typically an order of magnitude smaller than those observed experimentally ${ }^{16}$. Experiments reveal that nanofabrication techniques, such as FIB milling, modify the structural state near the surface in a manner that enhances tensile ductility ${ }^{16,17,21}$. However, this does not explain the observed size dependent transition in nanoscaled specimens fabricated using electroplating and not exposed to ion irradiation ${ }^{16}$. Recently, Zhao et al. proposed a statistically-informed theory that considers the effects of surface and bulk characteristics on size dependent macroscopic responses in MGs through the notion of extended defects $^{22}$. Their work makes broad connections to some of the observed experimental phenomena, but does not explain the mechanics of a size-dependent transition in the deformation mode of MGs.

No clear mechanism exists that can both explain the SB-necking transition in monolithic nanosized MGs and reconcile its apparent insensitivity to both the composition and the extent of ion damage. The question remains - what physical mechanism related to the dimensionality of MGs suppresses catastrophic failure? Tensile ductility of monolithic BMGs has been shown to increase in the presence of external notches, presumably as a result of the local stress triaxiality ${ }^{7}$, 8,23 . Sample synthesis unavoidably leads to finite surface roughness of different levels. The 
surface roughness of nanoscale samples is much higher relative to the sample size than that in most macroscale experiments. We speculate that it is the high surface roughness-to-diameter ratio in nanoscale samples that leads to similar notch-induced ductility observed in macroscale samples ${ }^{4}$. The magnitude and geometric characteristics of roughness differ among the various fabrication techniques. During mechanical straining, the topological surface features likely act as stress concentrators, similar to external notches ${ }^{24}$. For example, the stress intensity factor for a surface with normally distributed random roughness is given by $K_{t}=1+2 \sqrt{2}(\bar{a} / \beta)$ where $\bar{a}$ is the RMS roughness and $\beta$ is the autocorrelation length, which is qualitatively similar to that produced by single or multiple notches ${ }^{24}$. Could this nanoscale roughness be partially responsible for the tensile necking in nanoscale MGs? What is the length scale associated with the transition from catastrophic shear banding to necking? Does such dependence span a wide range of length scales? We employ large-scale MD simulations to investigate the effect of roughness on the BDT of MG nanopillars. The results reveal that small, densely populated, surface imperfections roughness play a critical role in determining the failure mode of MGs at the nanoscale. The results of this work suggest that the BDT in deformation of MGs may be valid at length scales well beyond the nanoscale, assuming the samples roughness-to-diameter ratio is above the intrinsic threshold value for the material.

The sample geometries were simulated to resemble those in experimental studies on nanoscale MGs. Figure 1a shows an atomic force microscope (AFM) image of a Ni-P MG surface exposed to FIB irradiation. It shows a valley-to-peak height difference in the 1-3 nm range over an area of $1 \mu \mathrm{m}^{2}$, with nanoscale ridges parallel to the direction at which the FIB was applied. In comparison, the scanning electron micrograph (SEM) of an electroplated Ni-P MG nanopillar with no FIB exposure indicates a nominally uniform surface along the pillar test section (Fig. S1-a in Supplementary Information). The pillar test section is noticeably smoother than the pillar cap due to the templated fabrication process discussed in the Methods section. Fig. S1-b shows an AFM image of Ni-P MG film surface generated by electrodeposition with no FIB exposure. The surface profile is diffuse with no well-defined peaks or valleys. Motivated by these observations, we performed a series of MD simulations on three closely related sets of cylindrical nanopillars under uniaxial tension: (i) smooth (S) specimens prepared by carving from larger bulk systems, (ii) single-notched (SN) specimens, which are identical to (i) with an intentional circumferential notch, and (iii) rough (R) specimens, which contain multiple densely populated circumferential notch-like imperfections placed along the specimen length. The simulations of SN specimens can help elucidate the role of roughness in the SB-necking transition and can be analyzed in terms of elasto-plastic fracture mechanics. In accordance with earlier experimental studies ${ }^{12,13,25}$ and the height profile shown in Fig. 1a, we introduced notches whose width and depth $(a)$ are equal to $1 \mathrm{~nm}$. For S and SN specimens, we systematically varied the specimen diameter in the $5 \leq d_{0} \leq 100 \mathrm{~nm}$ range while keeping the notch size fixed. For $\mathrm{R}$ specimens, we considered two specimen diameters, $d_{0}=15$ and $25 \mathrm{~nm}$, and varied the notch sizes (a) for each specimen to achieve the desired maximum notch size to diameter ratio. Circumferential notches were introduced with a maximum characteristic dimension in the range $0 \leq a \leq 2 \mathrm{~nm}$ and random notch spacing in the range $3 a \leq h \leq 5 a$ (Fig. 1b). In all the cases, the initial specimen length to diameter ratio was fixed at 2.5 .

Figures 2 and 3 highlight the main results of this work. They compare the evolution of the plastic deformation in S (Figs. 2a-f), SN (Figs. 2g-k), and R specimens (Figs. 3a-e). In these 
figures, the atoms are colored according to the local atomic shear $\operatorname{strain}^{26}\left(\varepsilon^{\text {Mises }}\right)$ and for clarity, only those with $\varepsilon^{\text {Mises }}>0.2$ are shown. The figures show the effect of surface imperfections on the manner in which strain localization occurs. Starting with the $\mathrm{S}$ specimens (Fig. 2a-f), we observe that SB is the characteristic deformation/failure mode in the diameter range $100 \mathrm{~nm} \geq$ $d_{0}>10 \mathrm{~nm}$. For $d_{0}=10 \mathrm{~nm}$ (Fig. 2e), we observe a mixed instability mode, which is governed by SB, but also exhibits some necking, resulting in a diffuse SB. For $d_{0}=5 \mathrm{~nm}$ (Fig. 2f) the specimen exhibits a shift in failure mode from SB to well-defined necking with no net shear. This transition occurs at nearly an order of magnitude smaller diameter $\left(d_{0}<\sim 10 \mathrm{~nm}\right)$ than those reported in experiments $9,10,11,16$.

The SN specimens (10 $\mathrm{nm} \leq d_{0} \leq 100 \mathrm{~nm}$ ) shown in Figs. $2 \mathrm{~g}$-k reveal a dramatic sizedependent trend in plastic deformation compared to their $S$ counterparts. For $d_{0}=100 \mathrm{~nm}$ (Fig. $2 \mathrm{~g}$ ), failure develops in the form of a sharp SB, akin to the corresponding $\mathrm{S}$ case (Fig. 2a). Comparing Figs. 2h-j (single-notched) to Figs. 2b-d (smooth surface), it is apparent that for $15 \leq$ $d_{0} \leq 50 \mathrm{~nm}$ the SN specimens exhibit a transition from SB to necking and the S specimens fail through formation and propagation of a localized SB. The $d_{0}=50 \mathrm{~nm} \mathrm{SN}$ specimen exhibits a diffuse SB with a much less pronounced shear offset compared to its smooth counterpart as well as to the $d_{0}=100 \mathrm{~nm} \mathrm{SN}$ specimen. At $d_{0}=25 \mathrm{~nm}$ the $\mathrm{SN}$ specimen shows a macroscopic instability governed by necking in the notched region with a minor shear offset, which indicates mixed mode failure dominated by necking. In the $15 \mathrm{~nm}$ SN nanopillar, a macroscopic instability and failure occur entirely through necking with no shear offset. These results clearly show that in nanoscale MGs the transition from SB to necking instabilities and failure occurs with decreasing diameter upon introduction of a single surface imperfection.

Figures 3 shows the evolution of the plastic deformation in R specimens of $d_{0}=25 \mathrm{~nm}$ for various maximum surface roughness amplitudes $(a)$. Notably, these results follow the same trend as those for the SN specimens. It can be seen that for roughness amplitude $a=0.25 \mathrm{~nm}$ (Fig. 3a), instability occurs in the form of a sharp SB. With increasing roughness amplitude, a clear transition from SB to necking is observed, akin to the SN specimens with increasing notch size. For this diameter, the transition occurs in the range $1 \leq a \leq 2 \mathrm{~nm}$. We also conducted a similar set of simulations for specimens with $d_{0}=15 \mathrm{~nm}$ (Fig. S2 in Supplementary Information), where a similar transition from SB to necking occurs in the $0.25 \leq a \leq 0.75 \mathrm{~nm}$ range.

In our simulations, $a$ is the characteristic scale of the surface imperfections, i.e. the notch size in $\mathrm{SN}$ specimens or the maximum roughness amplitude in $\mathrm{R}$ specimens. For $\mathrm{S}$ specimens simulated in this work, surface roughness amplitude is on the order of atomic structure, $a \sim 0.2$ $\mathrm{nm}$. The results clearly reveal a transition from SB to necking, which occurs both in the rougher samples with a fixed $d_{0}$ and in the smaller samples with a constant surface roughness, $a$. The dependence of the transition on both the surface roughness and the nanopillar diameter suggests that it is a combination of these parameters that governs failure in nanosized MGs, and can be represented as a roughness ratio, $\xi=a / d_{0}$. The threshold roughness ratio, $\xi_{t}$, appears to be in the range of $0.04 \leq \xi \leq 0.05$ based on Figs. 2, 3 and S2.

A more quantitative measure of necking is the true radial strain, $\eta=\ln \left(d / d_{0}\right) \approx$ $\left(d-d_{0}\right) / d_{0}$, where $d$ is the minimum cross-section diameter and $d_{0}$ is the original diameter of the 
sample. A quantitative measure of the degree to which shear bands contribute to the plastic strain is the ratio of the shear offset $\delta$ to the original sample diameter, $\varphi=\delta / d_{0}$ (inset in Fig. 4). Figure 4 shows these localization indices as a function of the roughness ratio $\xi$ at a fixed engineering strain $(\varepsilon=0.1)$ and it reveals a clear SB-necking transition at $\xi_{t} \sim 0.04$. The data in Fig. 4 accounts for all the MD simulations performed on $\mathrm{S}, \mathrm{SN}$, and $\mathrm{R}$ nanopillars. This threshold aspect ratio found from the simulations is consistent with the reported brittle (SB) to ductile (necking) transition size range reported in independent experimental investigations on MGs, both on the nano- and macro-scales, as shown in Fig. 5. A noteworthy feature of the present results is that the roughness-induced transition at $\xi_{t} \sim 0.04$ applies equally well to $\mathrm{S}$ specimens where the roughness is only associated with the discreteness of the amorphous atomic structure (Fig. 2a-f). Based on this latter observation, we should expect a SB-induced brittle failure at any diameter if the surface roughness could theoretically be completely eliminated (i.e., $a \approx 0 \mathrm{~nm}$ ). In fact, recent MD simulations on MG specimens prepared by simulated casting showed failure via catastrophic shear under tensile loading even for $d_{0}<5 \mathrm{~nm}$, consistent with the findings in this work $^{20}$. Necking would be expected for $\xi \geq \xi_{t}$, which is confirmed in both, nanoscale ${ }^{27,28}$ and macroscale specimens ${ }^{4,7}$. We postulate that it is this critical roughness ratio that unifies observations from diverse experiments and simulations by placing them in a context that is independent of absolute sample sizes.

Necking can result from conjugate SBs forming a symmetric slip mode. The transition from a SB instability to a necking instability for $\xi \geq \xi_{t}$ may be explained by theoretical analysis of the MD simulations results, which indicate that incipient localization occurs in the form of conjugate embryonic shear bands (ESBs) for all $\xi$ (see movies M1-M9 in the Supplementary Material). For $\xi<\xi_{t}$, only one of these ESBs develops into a runaway SB while further evolution of the other band is suppressed. For $\xi \geq \xi_{t}$ conjugate bands continue to co-evolve, creating a more widely spread SB that carries a substantial plastic strain in a diffuse volume that favors necking. In both scenarios, the microscopic picture that emerges is that STZs nucleate and incubate along a plane and collectively evolve to grow the SB volume. This phenomenological model is consistent with the shear plane criterion in that yield events are characterized by shearing on a plane over a distance where the yield stress is exceeded ${ }^{29}$.

The mechanics of a stress concentrator, i.e. a single notch or roughness, is characterized by (i) a heterogeneous stress state ahead of the stress concentrator that decays as the square root of the distance from the root and (ii) enhanced stress triaxiality, $T=\sigma_{h} / \sigma_{e}$, where $\sigma_{h}=$ $\operatorname{Tr}(\boldsymbol{\sigma}) / 3$ is the local mean hydrostatic stress $(\operatorname{Tr}(\boldsymbol{\sigma})$ is the trace of the stress tensor $\boldsymbol{\sigma})$ and $\sigma_{e}=$

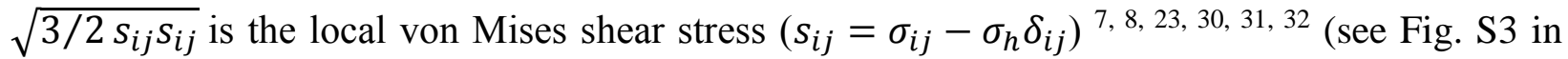
Supplementary Information). Occurrence of SBs ahead of the stress concentrators in our MD simulations implies that plasticity occurs because locally $\sigma_{e}>\sigma_{y}$, where $\sigma_{y}$ is the uniaxial yield strength. Continuum plasticity asserts that the material within a SB experiences a higher tensile triaxiality compared to the region outside the band $^{30,33}$. In the case of MGs, high tensile triaxiality implies enhanced free volume ${ }^{8}$ and void nucleation ${ }^{33}$ (dilatational plasticity). Based on these arguments, the fraction $f=V_{\mathrm{SB}} / V$ of the total $\mathrm{SB}$ volume $\left(V_{\mathrm{SB}}\right)$ to the specimen volume $(V)$ becomes an important quantity for describing the conditions for the SB (shear plasticity) to necking (dilatational plasticity) transition. 
Associated with the SB volume are the SB thicknesses, lengths of individual SBs and the number of SBs in a given region. While individual SB thicknesses in most MGs are nearly constant and roughly $\sim 10 \mathrm{~nm}^{28}$, the SB length scale may be related to effective roughness size (a) via the so-called plastic zone size $l_{p} \sim\left(K_{\mathrm{I}} / \sigma_{y}\right)^{2}$, where $K_{\mathrm{I}}=\sigma_{\infty} \sqrt{s \pi a}$ is the mode-I stress intensity factor and $s \sim 1-2$ depends on the specimen geometry ${ }^{34}$. Given that $K_{\mathrm{I}} \sim \sqrt{a}$, we may write $l_{p} \sim \alpha a$, which implies that the larger the roughness amplitude, the larger the plastic zone ahead of the stress concentration at a given load level is, thereby setting up the extent of individual SBs. The scaling parameter $\alpha$ (which may be thought of as the normalized plastic zone size) increases nonlinearly with increasing normalized remote stress $\left(\sigma_{\infty} / \sigma_{y}\right)$, which is also a function of the specimen and notch geometry, and may be described by $\alpha=A /\left(K_{t}\right)^{n}$ where $K_{t}$ is the appropriate stress concentration factor, $n \sim 2$ and $A$ is a constant. In the regime of interest ( $3 \geq K_{t} \geq 1$ ), a reasonable estimate of $\alpha$ for non-hardening materials is $3 \leq \alpha \leq 5^{34}$. Further, as incipient yield occurs at the notch root, where the stress is maximum $\left(\sigma_{\max }=K_{t} \sigma_{\infty}\right)$, we have $\sigma_{\infty} / \sigma_{y} \approx 1 / K_{t}$. Then, the roughness ratio becomes $\xi=a / d_{0}=l_{p}\left(K_{t}\right)^{n} / A d_{0}$.

In MG specimens exposed to FIB (Fig. 1c), $a \sim 1-3 \mathrm{~nm}$, which gives $l_{p}$ in the range $3-$ $15 \mathrm{~nm}$. Experiments indicate that the transition occurs at $d_{t} \sim 75-100 \mathrm{~nm}$. Assuming $l_{p} \approx 10 \mathrm{~nm}$ and $\alpha=3$, we obtain $\xi_{t}=\left(l_{p} / \alpha d_{t}\right) \approx 0.03-0.04$, which is remarkably close to the predictions from our MD simulations, where $\xi_{t}=0.04$. For nanoscale specimens the magnitude of $l_{p}$ is comparable to the incubation length scale, $l_{\text {inc }}$, proposed by Li and coworkers ${ }^{35}$ that describes the minimum length necessary for an ESB to propagate into a runaway SB. This comparison may be rationalized by expressing $l_{\text {inc }}=\left[\left(K_{c}\right)_{\text {inc }}^{2} / \tau_{\text {glue }}^{2}\right]$, where $\tau_{\text {glue }}$ is the quasi steady-state glue traction and $\left(K_{c}\right)_{i n c}=k c_{v}\left(T_{g}-T_{e n v}\right) / \sqrt{c_{s}}$ defined in terms of the thermal diffusivity $(k)$, volumetric specific heat $\left(c_{v}\right)$, glass transition temperature $\left(T_{g}\right)$, ambient temperature $\left(T_{\text {env }}\right)$ and shear wave speed $\left(c_{S}\right)$. In this limit, $l_{p}$ may be viewed as the critical embryonic plastic zone size in nanoscale specimens. Assuming that the SB thickness is proportional to $l_{p}$, it can be shown that $f=N \xi^{3} \alpha^{3} / \lambda$, where $N$ is the total number of SBs the ahead of the critical notch (usually $1 \leq N \leq 10$ ) and $\lambda=L_{0} / d_{0}$ is the specimen aspect ratio. Then, for the SB-necking transition the threshold SB volume $\left(V_{S B}\right)_{t}$ ahead of the critical notch is $\sim 10^{-3} \mathrm{~V}$.

This analysis shows that the threshold roughness ratio $\left(\xi_{t}\right)$ is governed by (i) the plastic zone size $\left(l_{p}\right)$ and (ii) the potency of the stress concentrators. For a given $d_{0}$ and $l_{p}$, $\xi_{t}$ will decrease with decreasing potency of the stress concentration. Results of this work imply that a specimen with a rough surface will transition from SB to necking at a larger diameter compared to the specimen with a single notch of the same size. The dependence of SB-necking transition on the (non-dimensional) roughness ratio suggests that it should apply over wide range of MG sample length scales, well beyond the nanoscale systems considered here.

Methods. We performed large-scale MD simulations on $\mathrm{Cu}_{64} \mathrm{Zr}_{36} \mathrm{MG}$ cylindrical nanowires with diameters $5 \leq d_{0} \leq 100 \mathrm{~nm}$ (at a fixed aspect ratio (length/diameter) of 2.5) using the MD code LAMMPS $^{36}$. The atomic interactions were computed using embedded atom method (EAM) potentials fitted to $\mathrm{CuZr}$ properties ${ }^{37}$. Time steps less than or equal to 5 fs were used in the integration of the equations of the motion. MG nanopillars were constructed by replication of a small $5.4 \times 5.4 \times 5.4 \mathrm{~nm}^{3}$ period $\mathrm{MG}$ sample generated with a procedure reported 
previously $^{26,38}$ (at a cooling rate of $10^{10} \mathrm{~K} / \mathrm{s}$ ). Nanopillars of different diameters were carved from a larger bulk MG system. Free surfaces were initially relaxed using Langevin dynamics for 0.02 ns. Residual stresses along the nanopillars axis (z-direction) were relaxed in an NPT ensemble for $0.02 \mathrm{~ns}$ prior to loading. Periodic boundary condition was applied along the $z$ direction (loading direction), while free surface conditions were maintained in the $x$ - and $y$ directions. During the loading, the temperature was maintained at $50 \mathrm{~K}$ using a Nosé-Hoover thermostat. Preliminary investigations of strain rate effects showed that strain rates up to $10^{8} \mathrm{~s}^{-1}$ do not have a strong effect on the failure mode of smooth surface samples which remains brittle failure by single, dominant SB propagation. Nonetheless, in all the simulations discussed in this work we adopted a nominal strain rate of $4 \times 10^{7} \mathrm{~s}^{-1}$. Engineering stress was calculated as the average of the atomic Virial stresses across the entire system. The generation and evolution of local inelastic deformation in the system was mined using the atomic local von Mises shear strain $^{26} \varepsilon_{i}^{\text {Mises }}$ and OVITO ${ }^{39}$ was employed for visualization.

The Ni-P MG was selected for the experimental studies because it can be fabricated via electrodeposition. The composition of this material was $\mathrm{Ni}_{73} \mathrm{P}_{23}$, as measured by energydispersive X-ray spectroscopy. The nanopillars shown in Fig. 1a were prepared using a templated electrodeposition ${ }^{40}$ process as described in detail elsewhere ${ }^{16}$. As can be observed in the figure, the roughness of the nanopillar test section is significantly less than that of the nanopillar cap. This occurs because the templated electrodeposition process utilized in fabricating these structures involves electrodepositing the Ni-P MG into a polymer template that contains vertical pores. The nanopillar test section forms within the smooth vertical pore of the polymer and thus has a roughness highly influenced by the polymer-MG interface, while the nanopillar cap forms outside of the pore and thus has an unrestrained roughness, similar to that of an electrodeposited film. Therefore the value of roughness measured on an electrodeposited film serves as an upper bound to the roughness of the actual nanopillar test section. Atomic force microscope (AFM) imaging was conducted on a $10 \mu \mathrm{m}$ thick Ni-P MG film surface generated by electrodeposition (with no FIB exposure) in order to determine this upper bound roughness. The resultant AFM image (Supplementary Fig. S2) shows a rather diffuse profile with no welldefined peaks or valleys, thus there is no directionality to the roughness of the electrodeposited MG. The upper bound value of roughness obtained for the electrodeposited film is quite high (Supplementary Fig. S2), but this is expected as the electrodeposited film is analogous to the cap of the electrodeposited nanopillar (Fig. 1a), which is noticeably rougher than the actual nanopillar test section.

To estimate the roughness of FIB-fabricated nanopillars (like those shown in Fig. 1b), we electrodeposited a film of Ni-P MG and applied analogous FIB conditions to those used for FIBfabricated nanopillars to a large area of the electrodeposited film. More specifically, we electrodeposited a $\sim 10 \mu \mathrm{m}$ thick $\mathrm{Ni}_{73} \mathrm{P}_{27} \mathrm{MG}$ film and FIB milled parallel to plane of the film utilizing a $30 \mathrm{keV} \mathrm{Ga}{ }^{+}$ion beam with the current sequentially reduced starting at $3 \mathrm{nA}$ then reducing to $1 \mathrm{nA}$ then $0.5 \mathrm{nA}$ then $100 \mathrm{pA}$ then $30 \mathrm{pA}$, and finally $10 \mathrm{pA}$ as is done with FIBfabricated nanopillars. The height profile was measured using an AFM, with the resultant image shown in Fig. 1c. As can be observed in Figure 1c, the FIB exposed surface has ridges parallel to the direction at which the FIB was applied and while the arithmetic roughness (absolute value of the deviation from the mean) was only $0.379 \mathrm{~nm}$, the characteristic roughness (peak to valley height difference) was in the 1-3 $\mathrm{nm}$ range. 
Acknowledgment. SA acknowledges financial support from A*STAR through the SINGA Award. RL and DZC acknowledge partial support from NSF Graduate Research Fellowships. This work was partially supported by the A*STAR Computational Resource Centre through the use of its high performance computing facilities.

\section{References}

1. Greer AL, Ma E. Bulk Metallic Glasses: At the Cutting Edge of Metals Research. Mrs Bulletin 32, 611-619 (2007).

2. Chen M. Mechanical Behavior of Metallic Glasses: Microscopic Understanding of Strength and Ductility. Annual Review of Materials Research 38, 445-469 (2008).

3. Schuh CA, Hufnagel TC, Ramamurty U. Mechanical behavior of amorphous alloys. Acta Materialia 55, 4067-4109 (2007).

4. Wang ZT, Pan J, Li Y, Schuh CA. Densification and Strain Hardening of a Metallic Glass under Tension at Room Temperature. Physical Review Letters 111, 135504 (2013).

5. Thamburaja P, Klusemann B, Adibi S, Bargmann S. The plastic yield and flow behavior in metallic glasses. Applied Physics Letters 106, 051903 (2015).

6. Hofmann DC, et al. Designing metallic glass matrix composites with high toughness and tensile ductility. Nature 451, 1085-1089 (2008).

7. Qu RT, Zhang QS, Zhang ZF. Achieving macroscopic tensile plasticity of monolithic bulk metallic glass by surface treatment. Scripta Materialia 68, 845-848 (2013).

8. Flores KM, Dauskardt RH. Mean stress effects on flow localization and failure in a bulk metallic glass. Acta Materialia 49, 2527-2537 (2001).

9. Guo H, et al. Tensile ductility and necking of metallic glass. Nature materials 6, 735-739 (2007). 
10. Jang D, Greer JR. Transition from a strong-yet-brittle to a stronger-and-ductile state by size reduction of metallic glasses. Nature materials 9, 215-219 (2010).

11. Tian L, Shan Z-W, Ma E. Ductile necking behavior of nanoscale metallic glasses under uniaxial tension at room temperature. Acta Materialia 61, 4823-4830 (2013).

12. Volkert CA, Donohue A, Spaepen F. Effect of sample size on deformation in amorphous metals. Journal of Applied Physics 103, 083539 (2008).

13. Luo JH, Wu FF, Huang JY, Wang JQ, Mao SX. Superelongation and Atomic Chain Formation in Nanosized Metallic Glass. Physical Review Letters 104, 215503 (2010).

14. Greer JR, De Hosson JTM. Plasticity in small-sized metallic systems: Intrinsic versus extrinsic size effect. Progress in Materials Science 56, 654-724 (2011).

15. Chen C, Pei Y, Kuzmin O, Zhang Z, Ma E, De Hosson J. Intrinsic size effects in the mechanical response of taper-free nanopillars of metallic glass. Physical Review B 83, 180201-180204 (2011).

16. Chen DZ, Jang D, Guan KM, An Q, Goddard WA, Greer JR. Nanometallic Glasses: Size Reduction Brings Ductility, Surface State Drives Its Extent. Nano Lett 13, 4462-4468 (2013).

17. Chen DZ, Gu XW, An Q, Goddard WA, Greer JR. Ductility and work hardening in nanosized metallic glasses. Applied Physics Letters 106, 061903 (2015).

18. Rys J, Valdevit L, Schaedler TA, Jacobsen AJ, Carter WB, Greer JR. Fabrication and Deformation of Metallic Glass Micro-Lattices. Adv Eng Mater 16, 889 (2014).

19. Li Q-K, Li M. Molecular dynamics simulation of intrinsic and extrinsic mechanical properties of amorphous metals. Intermetallics 14, 1005-1010 (2006).

20. Shi Y. Size-independent shear band formation in amorphous nanowires made from simulated casting. Applied Physics Letters 96, 121909 (2010).

21. Magagnosc DJ, Kumar G, Schroers J, Felfer P, Cairney JM, Gianola DS. Effect of ion irradiation on tensile ductility, strength and fictive temperature in metallic glass nanowires. Acta Materialia 74, 165-182 (2014). 
22. Zhao P, Li J, Wang Y. Extended defects, ideal strength and actual strengths of finitesized metallic glasses. Acta Materialia 73, 149-166 (2014).

23. Qu RT, Calin M, Eckert J, Zhang ZF. Metallic glasses: Notch-insensitive materials. Scripta Materialia 66, 733-736 (2012).

24. Medina HE, Pidaparti R, Hinderliter B. Celebrating the 100th Anniversary of Inglis Result: From a Single Notch to Random Surface Stress Concentration Solutions. Applied Mechanics Reviews 67, 010802 (2014).

25. Schuster BE, et al. Bulk and microscale compressive properties of a Pd-based metallic glass. Scripta Materialia 57, 517-520 (2007).

26. Adibi S, Branicio PS, Zhang Y-W, Joshi SP. Composition and grain size effects on the structural and mechanical properties of $\mathrm{CuZr}$ nanoglasses. Journal of Applied Physics 116, - (2014).

27. Sha ZD, Pei QX, Liu ZS, Zhang YW, Wang TJ. Necking and notch strengthening in metallic glass with symmetric sharp-and-deep notches. Sci Rep 5, 10797 (2015).

28. Zhang Y, Greer AL. Thickness of shear bands in metallic glasses. Applied Physics Letters 89, 071907 (2006).

29. Packard CE, Schuh CA. Initiation of shear bands near a stress concentration in metallic glass. Acta Materialia 55, 5348-5358 (2007).

30. Henann DL, Anand L. Fracture of metallic glasses at notches: Effects of notch-root radius and the ratio of the elastic shear modulus to the bulk modulus on toughness. Acta Materialia 57, 6057-6074 (2009).

31. Greer AL, Cheng YQ, Ma E. Shear bands in metallic glasses. Materials Science and Engineering: R: Reports 74, 71-132 (2013).

32. Wang WH. The elastic properties, elastic models and elastic perspectives of metallic glasses. Progress in Materials Science 57, 487-656 (2012). 
33. Murali P, Narasimhan R, Guo TF, Zhang YW, Gao HJ. Shear bands mediate cavitation in brittle metallic glasses. Scripta Materialia 68, 567-570 (2013).

34. Rice JR. Mechanics of crack tip deformatin and extention by fatigue. Fatigue Crack Propagation, ASTM STP 415, American Society of Testing Materials, 247 (1967).

35. Shimizu F, Ogata S, Li J. Yield point of metallic glass. Acta Materialia 54, 4293-4298 (2006).

36. Plimpton S. Fast Parallel Algorithms for Short-Range Molecular-Dynamics. J Comput Phys 117, 1-19 (1995).

37. Cheng YQ, Cao AJ, Sheng HW, Ma E. Local order influences initiation of plastic flow in metallic glass: Effects of alloy composition and sample cooling history. Acta Materialia 56, 5263-5275 (2008).

38. Adibi S, Sha Z-D, Branicio PS, Joshi SP, Liu Z-S, Zhang Y-W. A transition from localized shear banding to homogeneous superplastic flow in nanoglass. Applied Physics Letters 103, 211905 (2013).

39. Stukowski A. Visualization and analysis of atomistic simulation data with OVITO-the Open Visualization Tool. Modelling and Simulation in Materials Science and Engineering 18, 015012 (2010).

40. Lin CS, Lee CY, Chen FJ, Li WC. Structural Evolution and Internal Stress of NickelPhosphorus Electrodeposits. Journal of The Electrochemical Society 152, C370-C375 (2005).

\section{List of Figures}

Figure 1. (a) Atomic Force Microscopy (AFM) image obtained using a Ni-P MG surface (thin film geometry) after exposure to FIB irradiation. The color bar in indicates surface height. The peak to valley height (roughness amplitude) is in the 1-3 nm range. Panel (b) shows sample geometries used in MD simulations: smooth specimens (S), single-notched ( $\mathrm{SN}$ ) specimens, and rough (R) specimens. SN and R specimens possess U-shaped notch(es) with characteristic dimension $a$ along the circumference. For $\mathrm{R}$ 
specimens, $a$ represents the maximum notch size in the sample and the notch spacing $(h)$ is a random number in the range $3 a-5 a$.

Figure 2 . Transition from shear band instability to necking instability as a function of roughness ratio $(\xi)$ in MG nanopillars with smooth (S: a-f) and single-notched (SN: g-k) surfaces. Atoms are colored according to the calculated local atomic von Mises shear strain as indicated by the scale bar, and only atoms with shear strain $\geq 0.2$ are shown. In all the cases, a nominal strain rate $\left(\dot{\varepsilon}=4 \times 10^{7} \mathrm{~s}^{-1}\right)$ is applied, except specimens with $d_{0}=100 \mathrm{~nm}(\mathrm{a}, \mathrm{g})$, which are deformed at $10^{8} \mathrm{~s}^{-1}$. Note: smooth surfaces have an atomic scale roughness due to the finite atom size of $a=0.2 \mathrm{~nm}$, while $a$ indicates the notch depth in the SN specimens.

Figure 3. Transition from shear band instability to necking instability as a function of roughness ratio $(\xi)$ in MG nanopillars with rough (R) surfaces $\left(\boldsymbol{d}_{\mathbf{0}}=\mathbf{2 5} \mathrm{nm}\right)$. The color rendering is the same as in Fig. 2. In all the cases, $\dot{\varepsilon}=4 \times 10^{7} \mathrm{~s}^{-1}$.

Figure 4. Localization indices $(\varphi, \eta)$ as a function of roughness ratio $\xi=\boldsymbol{a} / \boldsymbol{d}_{\mathbf{0}}$ at fixed strain $(\boldsymbol{\varepsilon}=$ 0. 1). The necking ratio $\boldsymbol{\eta}=\ln \left(\boldsymbol{d} / \boldsymbol{d}_{\mathbf{0}}\right)$ and the normalized shear offset $\boldsymbol{\varphi}=\boldsymbol{\delta} / \boldsymbol{d}_{\mathbf{0}}$ are respectively defined in terms of the diameter $\boldsymbol{d}$ in the localized region and shear offset $\boldsymbol{\delta}$, and the initial diameter $\boldsymbol{d}_{\mathbf{0}}$. These indices clearly indicate a transition from shear banding to necking at $\boldsymbol{\xi} \sim \mathbf{0 . 0 4}$.

Figure 5. Failure mode dependence on the roughness ratio $\xi=a / d_{0}$ from the reported literature and current work. Mixed failure behavior indicates co-existence of SB-like and necking-like phenomena. Horizontal lines indicate a range of $\xi$ used in the experiments. 


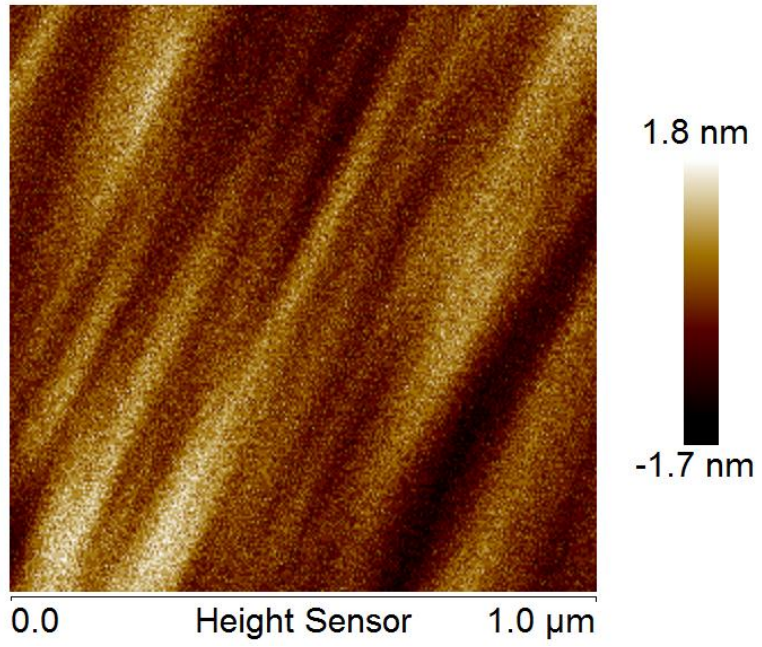

(a)
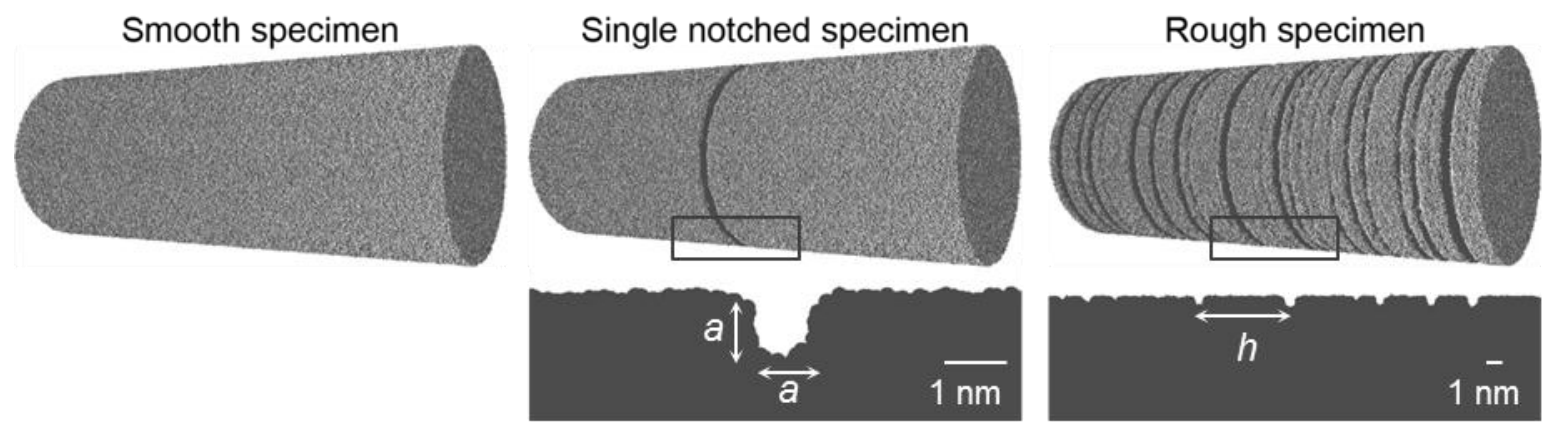

(b)

Figure 1. (a) Atomic Force Microscopy (AFM) image obtained using a Ni-P MG surface (thin film geometry) after exposure to FIB irradiation. The color bar in indicates surface height. The peak to valley height (roughness amplitude) is in the 1-3 nm range. Panel (b) shows sample geometries used in MD simulations: smooth specimens (S), single-notched (SN) specimens, and rough (R) specimens. SN and R specimens possess $\mathrm{U}$-shaped notch(es) with characteristic dimension $a$ along the circumference. For $\mathrm{R}$ specimens, $a$ represents the maximum notch size in the sample and the notch spacing $(h)$ is a random number in the range $3 a-5 a$. 


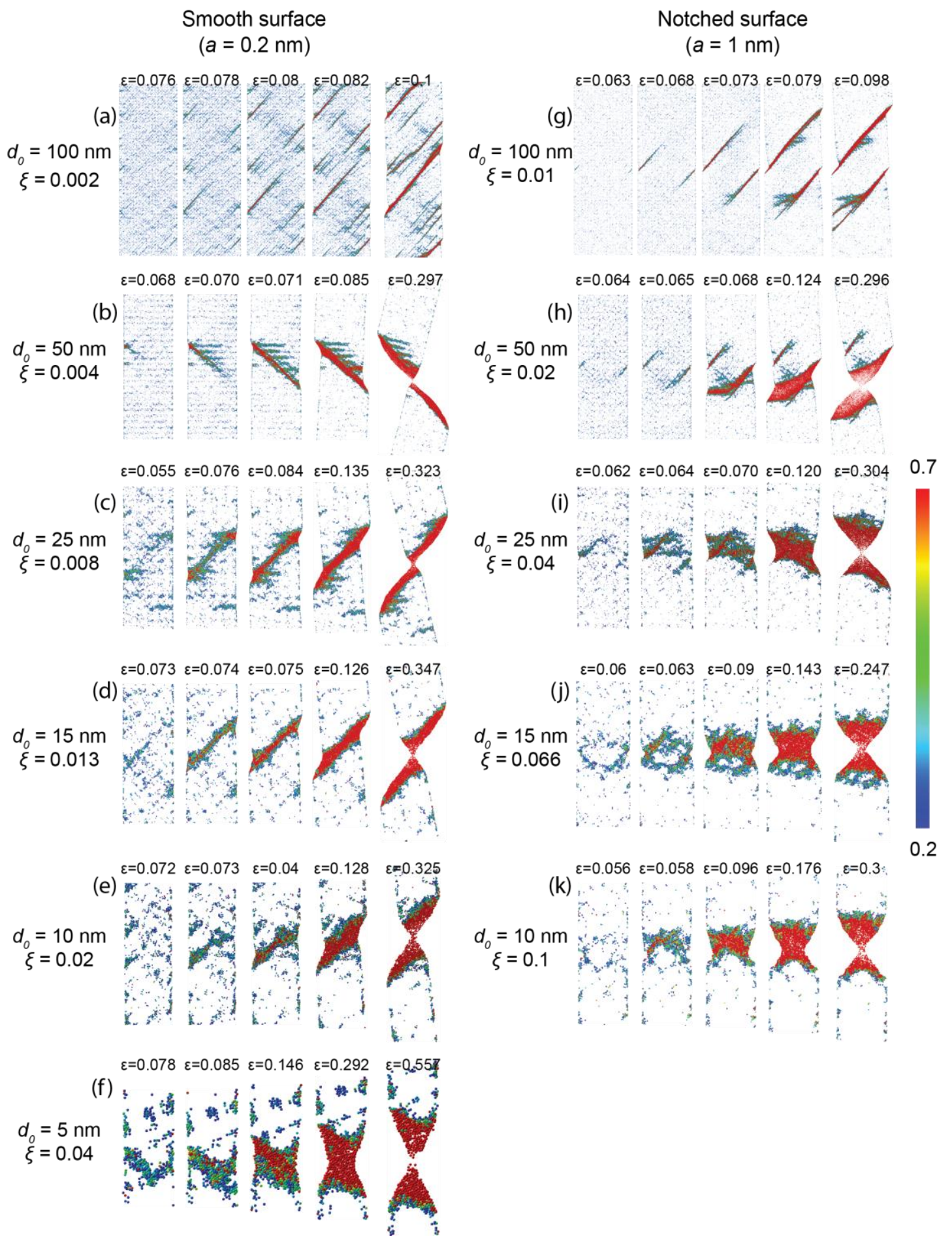

Figure 2. Transition from shear band instability to necking instability as a function of roughness ratio $(\xi)$ in MG nanopillars with smooth (S: a-f) and single-notched (SN: g-k) surfaces. Atoms are colored according to the calculated local atomic von Mises shear strain as indicated by the scale bar, and only atoms with shear strain $\geq 0.2$ are shown. In all the cases, a nominal strain rate $\left(\dot{\varepsilon}=4 \times 10^{7} \mathrm{~s}^{-1}\right)$ is applied, except specimens with $d_{0}=100 \mathrm{~nm}(\mathrm{a}, \mathrm{g})$, which are deformed at $10^{8} \mathrm{~s}^{-1}$. Note: smooth surfaces have an atomic scale roughness due to the finite atom size of $a=0.2 \mathrm{~nm}$, while $a$ indicates the notch depth in the SN specimens. 


\section{Rough Surface \\ $\left(d_{0}=25 \mathrm{~nm}\right)$}
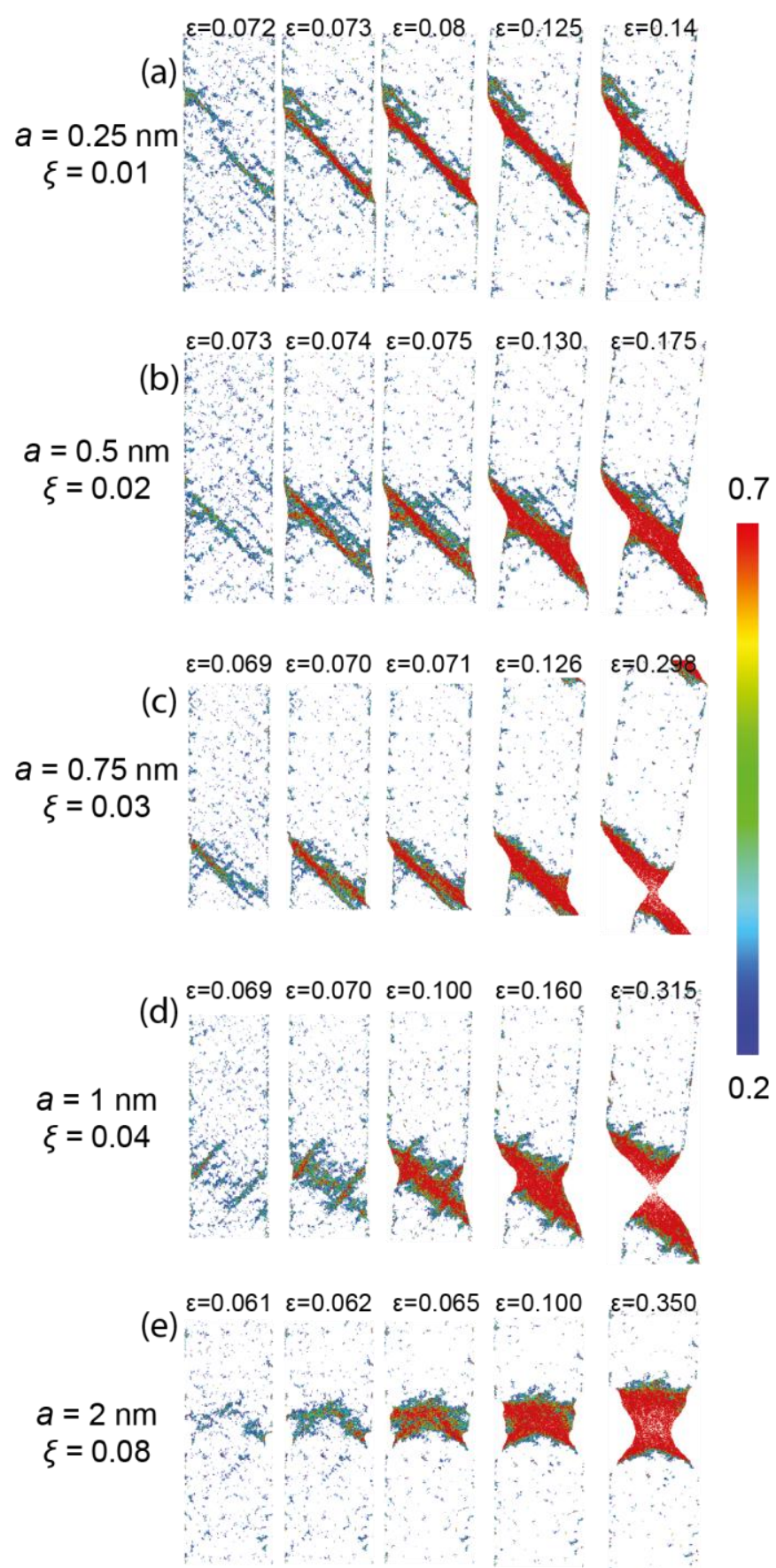

Figure 3. Transition from shear band instability to necking instability as a function of roughness ratio $(\xi)$ in MG nanopillars $\left(d_{0}=25 \mathrm{~nm}\right)$ with rough $(\mathrm{R})$ surfaces. The color rendering is the same as in Fig. 2 . In all the case, $\dot{\varepsilon}=4 \times 10^{7} \mathrm{~s}^{-1}$. 


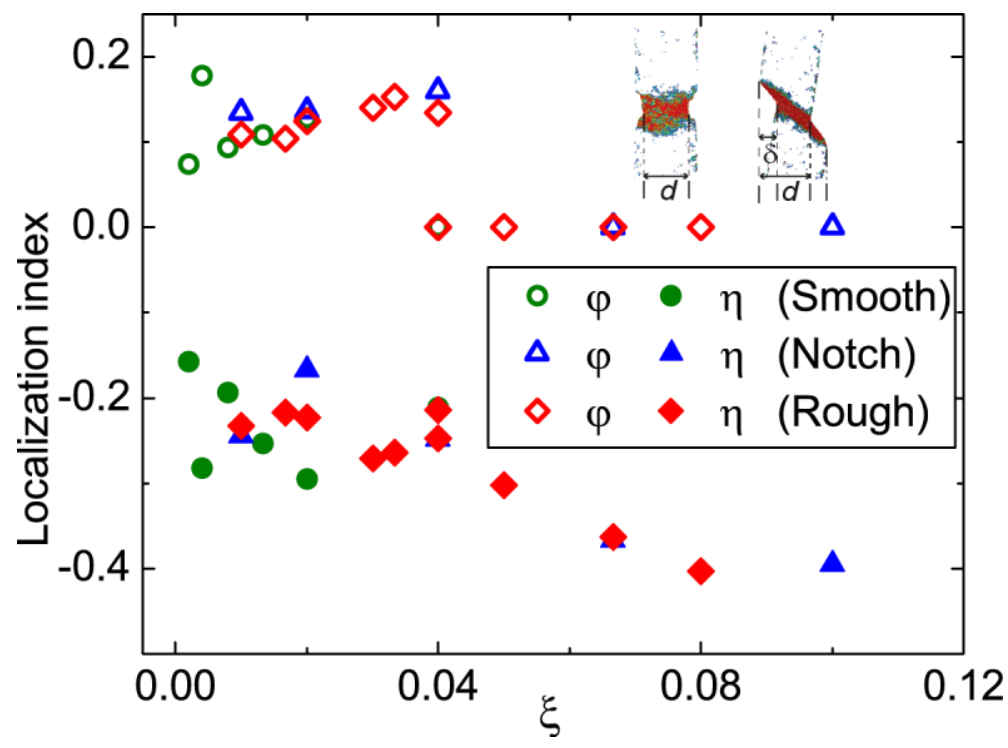

Figure 4. Localization indices $(\varphi, \eta)$ as a function of roughness ratio $\xi=a / d_{0}$ at fixed strain $(\varepsilon=0.1)$. The necking ratio $\eta=\ln \left(d / d_{0}\right)$ and the normalized shear offset $\varphi=\delta / d_{0}$ are respectively defined in terms of the diameter $d$ in the localized region and shear offset $\delta$, and the initial diameter $d_{0}$. These indices clearly indicate a transition from shear banding to necking at $\xi \sim 0.04$. 


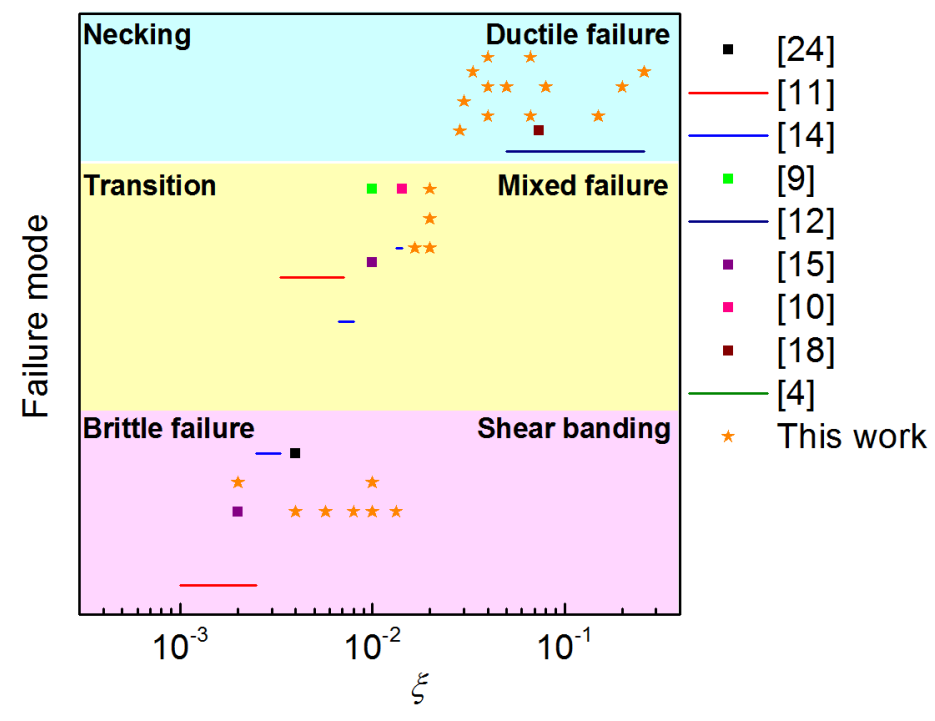

Figure 5. Failure mode dependence on the roughness ratio $\xi=a / d_{0}$ from the reported literature and current work. Mixed failure behavior indicates co-existence of SB-like and necking-like phenomena. Horizontal lines indicate a range of $\xi$ used in the experiments. 